\title{
Hypoxemia During One-Lung Ventilation: Does It Really Matter?
}

\author{
Chris Durkin $^{1} \cdot$ Kali Romano $^{1} \cdot$ Sinead Egan ${ }^{1} \cdot$ Jens Lohser ${ }^{1}$ \\ Accepted: 10 June 2021 / Published online: 7 July 2021 \\ (C) The Author(s), under exclusive licence to Springer Science+Business Media, LLC, part of Springer Nature 2021
}

\begin{abstract}
Purpose of Review Hypoxemia during one-lung ventilation, while decreasing in frequency, persists as an intraoperative challenge for anesthesiologists. Discerning when desaturation and resultant hypoxemia correlates to tissue hypoxia is challenging in the perioperative setting and requires a thorough understanding of the physiology of oxygen delivery and tissue utilization.

Recent Findings Oxygen delivery is not directly correlated with peripheral oxygen saturation in patients undergoing one-lung ventilation, emphasizing the importance of hemoglobin concentration and cardiac output in avoiding tissue hypoxia. While healthy humans can tolerate acute hypoxemia without long-term consequences, there is a paucity of evidence from patients undergoing thoracic surgery. Increasingly recognized is the potential harm of hyperoxic states, particularly in the setting of complex patients with comorbid diseases.

Summary Anesthesiologists are left to determine an acceptable oxygen saturation nadir that is individualized to the patient and procedure based on an understanding of oxygen supply, demand, and the consequences of interventions.
\end{abstract}

Keywords Hypoxemia $\cdot$ Hypoxia $\cdot$ Thoracic anesthesia $\cdot$ Oxygen delivery $\cdot$ One-lung ventilation

\section{Introduction}

One-lung ventilation, facilitated by either a double-lumen tube or bronchial blocker, is utilized to exclude ventilation to the operative thorax in a variety of cardiothoracic surgical procedures [1]. It results in an obligatory shunt as perfusion is maintained to the nonventilated lung. Although hypoxic pulmonary vasoconstriction (HPV) redirects a portion of this shunt to the ventilated side [2] and modern anesthesia agents impair HPV to a lesser degree [2], 4-10\% of patients still

This article is part of the Topical Collection on Thoracic Anesthesia

Jens Lohser

jens.lohser@vch.ca

Chris Durkin

christopher.durkin@vch.ca

Kali Romano

kali.romano@vch.ca

Sinead Egan

sineadegan311@gmail.com

1 Department of Anesthesiology, Pharmacology and Therapeutics, University of British Columbia, Vancouver General Hospital, JPP3 Room 3400, 899 West 12th Avenue, Vancouver, British

Columbia V5Z-1M9, Canada experience an oxygen saturation of less than $90 \%$ [3, 4]. In general, this level of desaturation triggers intervention due to a concern that organ cellular function may be impaired or injured by the reduction in oxygen delivery [5]. Frequently, the intervention includes an increase in inspired fraction of oxygen followed by a recruitment maneuver and escalation of positive end expiratory pressure (PEEP) to the ventilated lung [6•]. If this fails to correct the peripheral oxygen saturation to a perceived acceptable level, then low-level continuous positive airway pressure (CPAP) may be applied to the operative lung [5]. Each of these corrective steps has potential negative consequences that may be overlooked due to the innate discomfort anesthesiologists feel with a low peripheral oxygen saturation. However, oxygen delivery is not solely dependent on saturation but rather must be considered in concert with hemoglobin level and more importantly cardiac output [7]. Thus, whether hypoxemia reflected through peripheral oxygen saturation results in hypoxia is patient dependent. This review will provide a brief discussion of the determinants of tissue hypoxia with a focus on oxygen delivery, a modifiable parameter, as well as characterizing the critical level at which anaerobic metabolism will ensue. We would like to challenge the commonly held belief that a peripheral oxygen saturation less than $90 \%$ universally requires intervention by extrapolating evidence from outside of the thoracic operating room [5]. We will also explore the potential downsides of targeting a 
higher oxygen saturation and finally suggest patient-specific targets incorporating emerging monitoring technology.

\section{Hypoxemia vs Hypoxia}

Understanding the difference between hypoxemia and hypoxia is imperative to the decision-making around the management of patients undergoing one-lung ventilation. Hypoxemia, traditionally defined as a peripheral oxygen saturation less than $90 \%$, is commonly a trigger for intervention, whether by increasing inspired fraction of oxygen, optimizing ventilation in the non-operative lung, or in select circumstances intervening on the operative lung. Hypoxemia is the result of low oxygen content in arterial blood, but does not necessarily correlate to oxygenation in the tissues of target organs. The presumption that desaturation, or hypoxemia, results in harm to the patient and always requires action by the anesthesiologist is therefore not correct. It is important to distinguish that hypoxia, which can be regional or systemic, is the deprivation of oxygen at the tissue level and can result from several pathophysiologic mechanisms broadly defined into oxygen delivery or cellular utilization. As will be addressed further in this review, correlating hypoxemia to tissue hypoxia is a complex, multifactorial assessment that requires understanding the pathophysiology and tailoring decisionmaking to individual patients and perioperative scenarios.

\section{Determinants of Hypox(em)ia During OLV}

Hypoxia is medically classified into five pathophysiologic conditions, comprehensively reviewed elsewhere [8•]. Hypoxemia as it pertains to OLV is largely attributable to shunt which improves with time so long as HPV is not impaired and remains fixed unless ancillary measures are taken or two-lung ventilation is resumed. Transport of oxygen in the blood is largely a function of hemoglobin (Hgb) concentration, hemoglobin saturation $\left(\mathrm{SaO}_{2}\right)$, and cardiac output (CO) [7]. A reduction in any of these will decrease oxygen delivery $\left(\mathrm{DO}_{2}\right)$ and may lead to target organ dysfunction. Regional variations in microvascular tone, tissue edema, and to a much lesser degree dissolved oxygen content $\left(\mathrm{PaO}_{2}\right)$ are also variables that influence whether transported oxygen is able to reach the mitochondria of target organs [8•]. While the above mentioned all contribute to oxygen transport, in examining the oxygen delivery $\left(\mathrm{DO}_{2}\right)$ equation, it becomes clear that cardiac output is of particular importance.

$\mathrm{DO} 2=\mathrm{CO} \times\left\{\left(1.31 \times \mathrm{Hgb} \times \mathrm{SaO}_{2} \times 0.01\right)+\left(0.0225 \times \mathrm{PaO}_{2}\right)\right\}$

Practically, this suggests that a decrease in hemoglobin concentration or its saturation can be compensated by an increase in cardiac output to a certain extent [9], while the opposite would not be true. So, rather than assume all patients need an oxygen saturation of greater than $90 \%$, our focus may be more productive targeting a saturation in the clinical context of the hemoglobin and cardiac output so that global oxygen delivery will exceed the demand. Further to this, modification of cardiac output may be a more appropriate target for intervention if ventilatory maneuvers will lead to injury or impair surgical progress. In addition to improving oxygen delivery, increasing cardiac output to normal or high normal levels may improve the peripheral oxygen saturation due to an increase in mixed venous oxygen content $[10,11]$.

Imperative to the discussion of optimizing $\mathrm{DO}_{2}$ is $\mathrm{VO}_{2}$ (oxygen consumption or metabolic demand) and the $\mathrm{VO}_{2} /$ $\mathrm{DO}_{2}$ ratio. In normal physiology, $\mathrm{DO}_{2}$ adjusts in accordance with $\mathrm{VO}_{2}$ to maintain a $\mathrm{VO}_{2} / \mathrm{DO}_{2}$ ratio of $0.2-0.3$, which correlates to $20-30 \%$ extraction of oxygen or a mixed venous saturation $\left(\mathrm{SvO}_{2}\right)$ of $70 \%$. When $\mathrm{DO}_{2}$ decreases, $\mathrm{VO}_{2}$ is initially maintained through increased extraction (reflected clinically by a decreased $\mathrm{SvO}_{2}$ ), until a critical $\mathrm{DO}_{2}$ threshold is reached at which point $\mathrm{VO}_{2}$ becomes supply dependent, and further oxygen extraction is not possible. It is at this point (oxygen extraction of 60 $70 \%$ ) when any further increase in oxygen demand or decrease in delivery results in tissue hypoxia [12-14]. Unfortunately, trending $\mathrm{SvO}_{2}$ requires excessively invasive access for standard thoracic procedures, and waiting for anaerobic metabolism to ensue in order to detect an elevation in lactate as a reflection of inadequate oxygen delivery could result in deleterious organ effects.

Previous work has found this critical transition to occur when delivery falls below a $2: 1$ ratio of $\mathrm{DO}_{2} / \mathrm{VO}_{2}$ [15]. Importantly, we know that the critical oxygen delivery threshold is less than $8 \mathrm{mlO}_{2} / \mathrm{kg} / \mathrm{min}$ [16] and may be as low as $3 \mathrm{mlO}_{2} / \mathrm{kg} / \mathrm{min}$ [17]. Others have suggested that $500 \mathrm{ml} / \mathrm{min}$ is adequate for avoiding tissue oxygen deprivation while under general anesthesia [18]. On the consumption side of the ratio, it has previously been shown that $\mathrm{VO}_{2}$ during thoracic surgery is approximately $130 \mathrm{ml} /$ min [10]. Since we can roughly calculate oxygen delivery for any given patient, this presents the opportunity to target a safe delivery to consumption ratio and provide individualized patient care. A previous study evaluating calculated oxygen delivery during one-lung ventilation identified $14 \%$ of patients with delivery below the threshold of $500 \mathrm{ml} / \mathrm{min}$ [19•]. Interestingly, oxygen delivery was not correlated with peripheral oxygen saturation in these patients (Fig 1), further emphasizing the significant role of cardiac output. Limitations of such a strategy relate to the assumptions of minimally invasive cardiac output monitors and the time-consuming efforts this requires in the dynamic, high-paced thoracic operating room where global assessment may not reflect regional differences. 


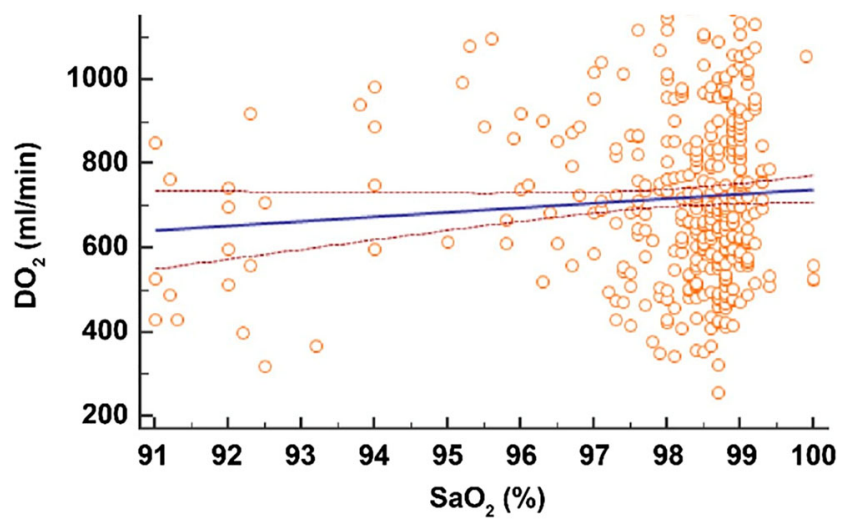

Figure $1 \mathrm{DO}_{2}$ was not correlated with $\mathrm{SaO}_{2}$ during OLV. Reproduced with permission from $\mathrm{Hahm}$ et al [19•].

\section{How Low Is Too Low?}

A paucity of data in thoracic surgery and the perioperative setting makes this a difficult question to address. There are no studies examining the tolerable level of hypoxemia during one-lung ventilation in human or animal models, or the consequence of transient intraoperative hypoxemia on outcomes such as surgical infection, renal insufficiency, myocardial dysfunction, or postoperative cognitive dysfunction. With the presence of electronic anesthesia record keeping now widely available, we may see this evaluated with retrospective data in the near future [20]. Nonetheless, hypoxemia has been identified as a common issue in all patients undergoing general anesthesia with $6.8 \%$ of patients experiencing a peripheral saturation less than $90 \%$ and $3.5 \%$ of patients having an event less than $85 \%$ for $2 \mathrm{~min}$ or longer [21]. This leaves us to infer clinical decisions based on data from physiologic and animal studies of acute hypoxemia, from human experiments with healthy volunteers exposed to hypoxic environments, and finally from observational work in critically ill patients with hypoxemia.

It is known from animal physiology experiments that tissue hypoxia is driven by $\mathrm{VO}_{2} / \mathrm{DO}_{2}$ mismatch, but there exists a margin of safety under normal physiologic circumstances such that $\mathrm{VO}_{2}$ is independent of $\mathrm{DO}_{2}$ through increased extraction until a critical $\mathrm{DO}_{2}$ is reached, at which point hypoxia will ensue $[13,22]$. The range at which this $\mathrm{VO}_{2} / \mathrm{DO}_{2}$ relationship exists is organ and context specific, with factors such as temperature, intercurrent illness, and even anesthetic agents influencing the ratio [13]. Anesthesia itself can reduce $\mathrm{VO}_{2}$ creating a larger margin of safety in the perioperative setting [23]. While this has enhanced our understanding of oxygen delivery, it does not evaluate the consequences of moderate hypoxemia exposure (80-90\%) that would mimic intraoperative conditions in the thoracic operating room. Animal models evaluating repercussions of hypoxemia have been focused on chronic disease states such as obstructive sleep apnea [24] or mixed hypoxia ischemia in the investigation of brain injury [25].
Healthy individuals exposed to hypoxic environments have been explored at length, particularly through studies on mountain climbers and high-altitude populations [26••]. With respect to the former where hypoxia progresses slowly over weeks, oxygen saturations in the sixties are observed without detrimental effects as a result of physiologic adaptations triggered by hypoxia-inducible factors including transcription of genes responsible for erythropoiesis and metabolic conditioning [8•], shifting of the oxygen dissociation curve, increase in cardiac output through tachycardia, and increased ventilation [27]. A more acute hypoxemia scenario is that of breath-hold divers who can experience saturation nadirs around $50 \%$ without appreciable consequence but again likely have some preconditioning [26••]. These two examples represent human adaptations that occur over time in healthy subjects, making it difficult to translate this understanding into the operating room. Interestingly, in the development of pulse oximeters more than 10,000 subjects were exposed to acute hypoxic conditions to promote hypoxemia detectable by pulse oximetry. An average study required approximately $15 \mathrm{~min}$ below a saturation of $80 \%$, often with brief periods as low as $45 \%$ [26••]. This did not result in detectable morbidity, but these studies were not designed to completely evaluate subjects' tolerance of hypoxemia but rather the reliability of pulse oximetry. Further reviews on the topic concluded that brief episodes of profound hypoxemia to a saturation of $50-70 \%$ in healthy humans are well tolerated without lasting consequences $[26 \bullet \bullet$. These levels are far below clinical comfort and acceptance during one-lung ventilation in patients in a setting of acute surgical stress and chronic comorbid conditions. However, with the suggestion that a mildly hypoxic environment may be an emerging therapy to treat of a variety of illness from mitochondrial disease [28] to spinal cord injury [29], data is signaling that short moderate $(80-90 \%$ saturation) hypoxic exposures are well tolerated in awake patients with a variety of comorbidities. Currently, a prolonged exposure study targeting a saturation of $80-85 \%$ is underway with an evaluation of the biochemical and physiological response [30].

The critical care environment is likely the most comparable to the intraoperative thoracic surgical environment. While several studies have been done comparing liberal to conservative oxygen therapy (targeting $\mathrm{SaO}_{2}$ 90-97\% vs $>97 \%$ ) [31••], randomizing patients to hypoxemia with a $\mathrm{SaO}_{2}$ less than $88 \%$ is of significant ethical concern. That being said, more than half of intensive care unit (ICU) admissions are a result of hypoxemic respiratory failure, some of which develop acute respiratory distress syndrome and hypoxemia of a comparable severity. While the severity of the hypoxemia may be similar, the etiology is quite distinct, with OLV hypoxemia largely attributable to intrapulmonary shunt. A large definitive randomized control trial is currently underway to evaluate the safety of lower saturation targets in critically ill patients, after 
an initial smaller feasibility study that assessed saturation values of $88-92 \%$ [32]. The premise of this work is based on the assumption that corrective measures may themselves be worse than permissive hypoxemia [33]. Certainly, the absence of harm has been shown in a smaller multicenter randomized controlled trial [34]. Strategies of permissive hypoxemia in the ICU, with $\mathrm{SaO}_{2} 80-90 \%$, are largely adapted from experience in patients with severe refractory acute respiratory distress syndrome requiring veno-venous extracorporeal membrane oxygenation (VV-ECMO).

In the setting of VV-ECMO, the peripheral arterial oxygen saturation is dependent on the proportion of venous blood flowing through the extracorporeal circuit compared to the venous blood shunting past the ECMO circuit to the right ventricle and intrapulmonary shunt [35]. This means that if cardiac output increases but VV-ECMO flow and pulmonary shunt remain unchanged, then peripheral oxygen saturation will decrease. The opposite also holds true in that an increase in peripheral oxygen saturation would be expected if the only variable altered is a reduction in cardiac output. Both of these scenarios assume no change in oxygen consumption. It is common that patients on VV-ECMO will have peripheral oxygen saturation of $80-85 \%$, which in the setting of adequate cardiac output and hemoglobin concentration to maintain a $\mathrm{VO}_{2} / \mathrm{DO}_{2}$ ratio of 0.3 does not result in harm and will meet the oxygen requirements of the body, as endorsed by the extracorporeal life support organization guidelines [36]. Elegant examples of this physiology are also reflected in studies by Spinelli and Bartlett [12]. In a review of registry data, patients on VV-ECMO with sustained saturations less than $90 \%$ have a similar mortality to those with a partial pressure of oxygen of 101-300 mmHg [37]. Although saturation targets vary amongst ECMO centers, permissive hypoxemia (saturation $85-90 \%$ ) is a frequent strategy to enable protective ventilation [38] and appears to have no long-term cognitive sequelae [39].

\section{Pitfalls of Targeting Higher Levels?}

If the interventions to correct hypoxemia during one-lung ventilation had no detrimental consequences then universal application would be expected. This is clearly not the case as we learn more about the negative effects of high inspired oxygen [40] and ventilation with harmful driving pressures $[41 \bullet \cdot]$. Additionally, as most oncologic thoracic surgery is now performed with a video-assisted approach [42], strategies relying on the operative lung are likely to impair surgical exposure and progress.

When faced with hypoxemia, a natural reflex is escalation of the fraction of inspired oxygen. We should consider this treatment strategy the same as increasing the dose of any medication, one that has benefits and risks [40]. High oxygen levels have been shown to reduce cardiac output through vasoconstricting properties [43], thereby potentially reducing oxygen delivery in a patient who may already be at a critical $\mathrm{VO}_{2} / \mathrm{DO}_{2}$ ratio. Prophylactically treating all patients with a high inspired oxygen level to avoid hypoxemia may also lead to significant hyperoxia as the majority of patients do not become hypoxemic during one-lung ventilation. This hyperoxia has been shown to impair microcirculation and may selectively impair cerebral and coronary blood flow, the latter leading to the recommendation that oxygen therapy in the treatment of myocardial infarction should not be routine $[8 \cdot, 44]$. Oxidative stress from high inspired oxygen levels to the ventilated lung is also a proposed contributor to lung injury after one-lung ventilation, the leading cause of death after thoracic surgery $[45 \cdot \bullet]$. Finally, hyperoxia can induce global inflammation and cell death as a result of the formation of reactive oxygen species that can easily overwhelm natural antioxidant mechanisms in the setting of systemic disease [8•].

Concurrent with a rise in inspired oxygen, it is often assumed that the dependent lung has become atelectatic. While appropriate recruitment and escalation of PEEP (open lung ventilation) are justified [46], anesthesiologists must avoid the temptation to increase ventilator parameters to levels known to be associated with lung injury [41•]. Tidal volumes above $4-6 \mathrm{ml} / \mathrm{kg}$ ideal body weight and driving pressures in excess of 14 should be avoided [43]. Refractory hypoxemia is assumed to be secondary to shunt through the collapsed lung and relative failure of hypoxic pulmonary vasoconstriction, which leads the anesthesiologist to turn their attention towards interventions on the operative lung. Unfortunately, any attempts at oxygenating the surgical lung may impair surgical progress with a video-assisted approach. This could prompt conversion to an open procedure, with its resultant increase in perioperative complications, length of stay [47], and possibly long-term mortality [48].

\section{Finding the Right Balance}

The search for the optimal range of intraoperative hemoglobin concentration, fluid management, and mean arterial blood pressure continues to occupy a significant amount of research efforts $[49,50 \bullet, 51]$. Intraoperative oxygen saturation deserves similar attention as there is experience to show that extremes, both too high and too low, may be deleterious. The difficulty comes in defining these margins, then anticipating and intervening to avoid significant exposure outside of the optimal range. In regard to the upper limit, newer pulse oximeters using multi-wavelength pulse co-oximetry have been proposed as a tool to monitor and optimize oxygen therapy [52]. They provide an oxygen reserve index (ORI) with a unitless range of 0 to 1 , which correlates with a partial pressure of oxygen between 100 and $200 \mathrm{mmHg}$ [53•]. This index could be used to avoid hyperoxia since the traditional pulse 
oximeter saturation greater than $97 \%$ correlates with a $\mathrm{PaO}_{2}$ anywhere between 90 and $600 \mathrm{mmHg}$. Additionally, a trending reduction in reserve index could be a signal to intervene prior to the development of significant hypoxemia. If unavailable, it would be worthwhile to titrate down inspired oxygen fraction to the point when saturation starts to decrease. Maintaining the inspired level just above this inflection would ensure that unnecessarily elevated $\mathrm{PaO}_{2}$ levels with their detrimental consequences are avoided.

There will still be a minority of patients who develop hypoxemia despite optimized anesthetic management. It is this situation where calculating that individual patients' oxygen delivery could be used to inform decision-making. If delivery is calculated to be twice that of critical oxygen consumption, it may be reasonable to assume that the patient can tolerate the level of desaturation to facilitate the operation, if the alternative is a potentially injurious or surgically prohibitive maneuver. It seems likely that many patients would tolerate a peripheral saturation between 85 and $90 \%$ without negative sequelae in the setting of adequate hemoglobin concentration and more importantly cardiac output. Difficulty arises with this proposal as the accurate evaluation of cardiac output outside of the traditional thermodilution method that requires a pulmonary artery catheter is uncertain. While minimally invasive methods relying on arterial line waveform analysis in addition to biometric and demographic data to estimate stroke volume for an individual patient are becoming more widely available [54], their effectiveness outside of goal-directed fluid therapy in terms of a true and precise reading compared to an invasive method has been questioned [54]. Additionally, the ability of these monitors to perform in the setting of onelung ventilation has shown discordance when compared to pulmonary artery catheter values [55]. This means that any calculation of oxygen delivery will be an estimate.

The combination of this information leaves the anesthesiologist to determine the optimal range of acceptable saturation for the specific patient with incomplete information. Ideally, we could evaluate oxygen delivery to critical organs selectively and continuously; however, this is not possible. The previously mentioned efforts to avoid hyperoxia should be taken while the lowest acceptable saturation remains uncertain. It is likely that most providers will continue to target a traditional saturation of greater or equal to $90 \%$.

\section{Conclusion}

Oxygen delivery in the anesthetized patient during one-lung ventilation is complex and is dependent on hemoglobin, saturation, and cardiac output. Oxygen delivery must exceed oxygen consumption or cellular hypoxia will occur. Reductions in oxygen transport are multiplicative, not additive, and desaturation in the context of anemia or low cardiac output can be detrimental. Intraoperatively, it is often the peripheral oxygen saturation component that garners the most attention. We know that healthy awake humans can tolerate transient hypoxemia without consequence, but there is a paucity of data from patients under general anesthesia or more specifically an absence of studies during one-lung ventilation. Extrapolating data from outside of the operating room, it seems plausible that transient mild (85-90\%) hypoxemia would be well tolerated when coexisting with adequate cardiac output and hemoglobin. Exact measurements of cardiac output during one-lung ventilation have not been validated with minimally invasive techniques making the calculation of oxygen delivery an estimate. When management steps to correct hypoxemia are noninjurious and do not impede surgical progress, such interventions should be performed. However, when anesthetic management has been optimized, the downside of further efforts needs to be balanced against those of permissive hypoxemia. This balance of risk will continue to be debated until further studies inform decision-making.

Author Contribution All authors prepared the manuscript and approved the final copy of the manuscript.

\section{Declarations}

Conflict of Interest The authors do not have any potential conflicts of interest to disclose.

Human and Animal Rights and Informed Consent This article does not contain any studies with human or animal subjects performed by any of the authors.

\section{References}

Papers of particular interest, published recently, have been highlighted as:

- Of importance

•- Of major importance

1. Campos JH. Progress in lung separation. Thorac Surg Clin. 2005;15(1):71-83.

2. Lumb AB, Slinger P. Hypoxic pulmonary vasoconstriction: physiology and anesthetic implications. Anesthesiology. 2015;122(4): 932-46.

3. Schwarzkopf K, et al. Oxygenation during one-lung ventilation: the effects of inhaled nitric oxide and increasing levels of inspired fraction of oxygen. Anesth Analg. 2001;92(4):842-7.

4. Guenoun T, Journois D, Silleran-Chassany J, Frappier J, D'Attellis N, Salem A, et al. Prediction of arterial oxygen tension during onelung ventilation: analysis of preoperative and intraoperative variables. J Cardiothorac Vasc Anesth. 2002;16(2):199-203.

5. Rozé H, Lafargue M, Ouattara A, Riou B. Case scenario: management of intraoperative hypoxemia during one-lung ventilation. Anesthesiology. 2011;114(1):167-74. 
6. Campos JH, Feider A. Hypoxia during one-lung ventilation-a review and update. J Cardiothorac Vasc Anesth. 2018;32(5):2330-8 Recent review article focusing on the factors that predict hypoxemia during OLV, physiology of OLV and the ventilatory strategies to improve oxygenation.

7. Dunn JO, Mythen MG, Grocott MP. Physiology of oxygen transport. BJA Education. 2016;16(10):341-8.

8. Nakane M. Biological effects of the oxygen molecule in critically ill patients. J Intensive Care. 2020;8(1):95 A major review article examining the negative effects of hyperoxia in critical care patients, the lack of evidence to support a liberal oxygen therapy strategy and advises a conservative approach with cautious monitoring.

9. Siebenmann C, Lundby C. Regulation of cardiac output in hypoxia. Scand J Med Sci Sports. 2015;25(Suppl 4):53-9.

10. Slinger P, Scott WA. Arterial oxygenation during one-lung ventilation. A comparison of enflurane and isoflurane. Anesthesiology. 1995;82(4):940-6.

11. Levin AI, Coetzee JF. Arterial oxygenation during one-lung anesthesia. Anesth Analg. 2005;100(1):12-4.

12. Spinelli E, Bartlett RH. Relationship between hemoglobin concentration and extracorporeal blood flow as determinants of oxygen delivery during venovenous extracorporeal membrane oxygenation: a mathematical model. ASAIO J. 2014;60(6):688-93.

13. Leach RM, Treacher DF. The pulmonary physician in critical care • 2: Oxygen delivery and consumption in the critically ill. Thorax. 2002;57(2):170-7.

14. van Beest P, Wietasch G, Scheeren T, Spronk P, Kuiper M. Clinical review: use of venous oxygen saturations as a goal - a yet unfinished puzzle. Crit Care. 2011;15(5):232.

15. Spinelli E, Bartlett RH. Anemia and transfusion in critical care: physiology and management. J Intensive Care Med. 2016;31(5): 295-306.

16. Lieberman JA, Weiskopf RB, Kelley SD, Feiner J, Noorani M, Leung $\mathrm{J}$, et al. Critical oxygen delivery in conscious humans is less than $7.3 \mathrm{ml} \mathrm{O} 2 \times \mathrm{kg}(-1) \times \mathrm{min}(-1)$. Anesthesiology. 2000;92(2): $407-13$.

17. Ronco JJ, Fenwick JC, Tweeddale MG, Wiggs BR, Phang PT, Cooper DJ, et al. Identification of the critical oxygen delivery for anaerobic metabolism in critically ill septic and nonseptic humans. Jama. 1993;270(14):1724-30.

18. Sykes O. Metabolic oxygen requirements. Anaesthesia. 2017;72(3):415-6.

19. Hahm, T.S., H. Jeong, and H.J. Ahn Systemic oxygen delivery during one-lung ventilation: comparison between propofol and sevoflurane anaesthesia in a randomised controlled trial. J Clin Med. 2019 8(9). Prospective randomised trial examining differences in $\mathrm{DO}_{2}$ between propofol and sevoflurane. Interesting secondary outcomes showing no correlation between $\mathrm{SaO}_{2}$ and $\mathrm{DO}_{2}$.

20. Dziadkowiec, O.D., Jeffery S.; Jayaraman Muralidharan, Vignesh; Novak, Megan L.; and Cornett, Brendon T, Improving the quality and design of retrospective clinical outcome studies that utilize electronic health records. HCA Healthcare Journal of Medicine, 2020. 1(3)

21. Ehrenfeld JM, Funk LM, van Schalkwyk J, Merry AF, Sandberg WS, Gawande A. The incidence of hypoxemia during surgery: evidence from two institutions. Can J Anaesth. 2010;57(10):888-97.

22. Cain SM. Oxygen delivery and uptake in dogs during anemic and hypoxic hypoxia. J Appl Physiol Respir Environ Exerc Physiol. 1977;42(2):228-34.

23. Van der Linden P, et al. Effects of anesthetic agents on systemic critical O2 delivery. J Appl Physiol (1985). 1991;71(1):83-93.

24. Farré, R., et al., Intermittent hypoxia severity in animal models of sleep apnea. Frontiers in Physiology, 2018. 9(1556).
25. Hamdy N, Eide S, Sun HS, Feng ZP. Animal models for neonatal brain injury induced by hypoxic ischemic conditions in rodents. Exp Neurol. 2020;334:113457.

$26 . \bullet$ Bickler PE, et al. Effects of acute, profound hypoxia on healthy humans: implications for safety of tests evaluating pulse oximetry or tissue oximetry performance. Anesth Analg. 2017;124(1):14653 This review on the effects of hypoxia on human physiology strongly suggests that unless the hypoxia is chronic or combined with circulation failure, that the effects of even profound hypoxia are reversible and do not lead to any long term sequellae in otherwise healthy individuals.

27. Botella de Maglia, J., R. Real Soriano, and L. Compte Torrero, [Arterial oxygen saturation during ascent of a mountain higher than 8,000 meters]. Med Int, 2008. 32(6): p. 277-281.

28. Jain IH, Zazzeron L, Goli R, Alexa K, Schatzman-Bone S, Dhillon $\mathrm{H}$, et al. Hypoxia as a therapy for mitochondrial disease. Science. 2016;352(6281):54-61.

29. Hayes HB, Jayaraman A, Herrmann M, Mitchell GS, Rymer WZ, Trumbower RD. Daily intermittent hypoxia enhances walking after chronic spinal cord injury: a randomized trial. Neurology. 2014;82(2):104-13.

30. Rezoagli, E., Massachusetts General Hospital, Harvard Medical School, Prolonged hypoxic breathing in healthy volunteers: a safety study (MGH-nitrogen). 2017-2022.

31.• Mackle D, et al. Conservative Oxygen Therapy during Mechanical Ventilation in the ICU. N Engl J Med. 2020;382(11):989-98 Largest ICU trial conducted in ventilated patients to date examining whether usual oxygen therapy versus a conservative oxygen strategy affected the number of ventilator free days. The authors found no difference in the number of ventilator free days in the first 28 days of ICU care.

32. Martin DS, Brew-Graves C, McCartan N, Jell G, Potyka I, Stevens $\mathrm{J}$, et al. Protocol for a feasibility randomised controlled trial of targeted oxygen therapy in mechanically ventilated critically ill patients. BMJ Open. 2019;9(1):e021674.

33. Martin DS, Grocott MP. Oxygen therapy in critical illness: precise control of arterial oxygenation and permissive hypoxemia. Crit Care Med. 2013;41(2):423-32.

34. Panwar R, Hardie M, Bellomo R, Barrot L, Eastwood GM, Young $\mathrm{PJ}$, et al. Conservative versus liberal oxygenation targets for mechanically ventilated patients. A pilot multicenter randomized controlled trial. Am J Respir Crit Care Med. 2016;193(1):43-51.

35. Zante B, Berger DC, Schefold JC, Bachmann KF. Dissociation of arterial oxygen saturation and oxygen delivery in VVECMO: the trend is your friend. J Cardiothorac Vasc Anesth. 2021;35(3):962-3

36. (ELSO), E.L.S.O., Guidelines for adult respiratory failure. 2017.

37. Munshi L, Kiss A, Cypel M, Keshavjee S, Ferguson ND, Fan E. Oxygen thresholds and mortality during extracorporeal life support in adult patients. Crit Care Med. 2017;45(12):1997-2005.

38. Romano TG, Mendes PV, Park M, Costa ELV. Extracorporeal respiratory support in adult patients. J Bras Pneumol. 2017;43(1): 60-70.

39. Lindén VB, et al. ECMO in ARDS: a long-term follow-up study regarding pulmonary morphology and function and health-related quality of life. Acta Anaesthesiol Scand. 2009;53(4):489-95.

40. Staehr-Rye AK, Meyhoff CS, Scheffenbichler FT, Vidal Melo MF, Gätke MR, Walsh JL, et al. High intraoperative inspiratory oxygen fraction and risk of major respiratory complications. Br J Anaesth. 2017;119(1):140-9.

41.• Neto AS, et al. Association between driving pressure and development of postoperative pulmonary complications in patients undergoing mechanical ventilation for general anaesthesia: a metaanalysis of individual patient data. Lancet Respir Med. 2016;4(4): 272-80 Landmark review paper examining over 2250 patients 
illustrating that high driving pressures intraoperatively led to increased postoperative pulmonary complications.

42. Sihoe AD. The evolution of minimally invasive thoracic surgery: implications for the practice of uniportal thoracoscopic surgery. J Thorac Dis. 2014;6(Suppl 6):S604-17.

43. Asfar P, Singer M, Radermacher P. Understanding the benefits and harms of oxygen therapy. Intensive Care Med. 2015;41(6): 1118-21.

44. Wijesinghe M, Perrin K, Ranchord A, Simmonds M, Weatherall M, Beasley R. Routine use of oxygen in the treatment of myocardial infarction: systematic review. Heart. 2009;95(3):198-202.

45.• Lohser J, Slinger P. Lung injury after one-lung ventilation: a review of the pathophysiologic mechanisms affecting the ventilated and the collapsed lung. Anesth Analg. 2015;121(2):302-18 Landmark review paper looking at the pathophysiology of lung injury following OLV and strategies to reduce it, given its recognition as the leading cause of death following thoracic surgery.

46. Gama de Abreu M, et al. Open-lung ventilation strategy during general anesthesia: what happens intraoperatively stays intraoperatively. Anesthesiology. 2020;133(5):982-4.

47. Nwogu CE, D'Cunha J, Pang H, Gu L, Wang X, Richards WG, et al. VATS lobectomy has better perioperative outcomes than open lobectomy: CALGB 31001, an ancillary analysis of CALGB 140202 (Alliance). Ann Thorac Surg. 2015;99(2):399-405.

48. Al-Ameri M, et al. Video-assisted thoracoscopic versus open thoracotomy lobectomy: a Swedish nationwide cohort study. J Thorac Dis. 2018;10(6):3499-506.

49. Hensley NB, Frank SM, Prochaska MT. Intraoperative Transfusion Targets: Avoiding the Extremes. Anesth Analg. 2019;129(3):642-3.
50. Miller TE, Myles PS. Perioperative fluid therapy for major surgery. Anesthesiology. 2019;130(5):825-32 A comprehensive review on intraoperative fluid management for major surgery, including criteria to guide therapy and suggestions on optimal volume and type of fluid.

51. Saugel B, Sessler DI. Perioperative blood pressure management. Anesthesiology. 2021;134(2):250-61.

52. Scheeren TWL, Belda FJ, Perel A. The oxygen reserve index (ORI): a new tool to monitor oxygen therapy. J Clin Monit Comput. 2018;32(3):379-89.

53. Vos, J.J., et al., Oxygen Reserve Index: Validation of a New Variable. Anesthesia \& Analgesia, 2019. 129(2). Small prospective trial showing a correlation between $\mathrm{PaO}_{2}$ and oxygen reserve index lending support to the use of $\mathrm{ORi}$ as a potential noninvasive monitor for trending oxygenation status in patients receiving supplemental oxygen.

54. Saugel B, Thiele RH, Hapfelmeier A, Cannesson M. Technological Assessment and objective evaluation of minimally invasive and noninvasive cardiac output monitoring systems. Anesthesiology. 2020;133(4):921-8.

55. Suehiro K, Tanaka K, Yamada T, Matsuura T, Mori T, Funao T, et al. The ability of the Vigileo-FloTrac system to measure cardiac output and track cardiac output changes during one-lung ventilation. J Clin Monit Comput. 2015;29(3):333-9.

Publisher's Note Springer Nature remains neutral with regard to jurisdictional claims in published maps and institutional affiliations. 